\title{
How are fifth metatarsal fractures managed by the virtual fracture clinic?
}

\author{
Zeenat Mirza, Anand Pillai and Mohamad Alqubaisi* \\ University Hospital of South Manchester NHS Foundation Trust, UK
}

\begin{abstract}
Background: A fifth metatarsal fracture is a frequent type of fracture that is not classified during assessment.

Aims: To analyse the patterns in management plans of patients that have presented with basal fifth metatarsal fractures to promote a change in the service provided.

Materials and methods: Study of 270 patients conducted over a period of 19 months classified the fractures using Dameron-Lawrence-Bofte classification, the Torg classification and the Stewart classification. Their data was then identified for treatment, non-union and delayed union.

Results: Out of the 270 patients that presented to the Virtual Fracture Clinic with fifth metatarsal fractures, $53.3 \%$ were basal fractures of the fifth metatarsal. Using the Dameron-Lawrence-Bofte classification it was found that $73.6 \%$ were Zone $1,22.2 \%$ were Zone 2 and $4.2 \%$ were Zone 3 . It is shown that there is a $57 \%$ chance of delayed union in Zone 1 and 43\% likelihood in Zone 2, where the treatment period is longer than 12 weeks. The highest occurrence of non-union is in Zone 2 of 57\%, 29\% in Zone 1 and 14\% in Zone 3. In this cohort, 4 patients required operative management, 3 of these were Zone 2 and 1 was Zone 1.
\end{abstract}

Conclusion: Zone 1 and 3 fractures require conservative management and could be discharged routinely with advice that it could take up to 12 weeks to heal. However, Zone 2 fractures are more likely to need a surgical approach and thus should receive a follow up appointment at 6 weeks.

\begin{abstract}
Abbreviations: UHSM: University Hospital of South Manchester NHS foundation Trust; VFC: Virtual Fracture Clinic; NHS: National Health Service; A\&E: Accident and Emergency; CT: Computed tomography; MRI: Magnetic Resonance Imaging.
\end{abstract}

\section{Introduction}

The technological developments within the National Health Service (NHS) provide a foundation to improve the quality of care and patient satisfaction without additional costs. This is attained by replacing the traditional clinic pathway for a virtual platform, where the consultations can occur with fewer resources and physicians. This audit has reviewed the efficiency of the Virtual Fracture Clinic (VFC) in University Hospital of South Manchester NHS foundation trust (UHSM) in managing fractures of the proximal fifth metatarsal. A significant difference is visible in the management of this type of fracture in particular if it is doubted to be a Jones' fracture, as the boundaries for this are ambiguous. Sir Jones' was the first to describe this type of fracture as a break at the "proximal $3 / 4$ segment of the shaft distal to the styloid" [1], now this is discussed as a fracture at the junction of the metaphysis and diaphysis without distal extension. It is significant to identify this type of fracture as it occurs in an area of rich blood supply thus an increased likelihood of leading to delayed union, non-union and difficulties in treatment [2]. A large proportion of such location are tuberosity related and have an excellent outlook as it is not concerning in relation to the anatomy (Zone 1, Dameron-LawrenceBofte Classification [3]) compared to those at metaphysis-diaphysis junction (Zone 2, Dameron-Lawrence-Bofte Classification [3]) or stress fractures at the diaphysis including the proximal $1.5 \mathrm{~cm}$ (Zone 3, Dameron-Lawrence-Bofte Classification [3]). (Figure 1). There are different ways to classify fractures including anatomically: base, shaft, neck and head (Figure 2) [4]; however, this does not guide towards a prognosis unlike the Torg Classification. The recommendations Torg et al's study makes for the management of these fracture is dependent on the fracture's radiological appearance and healing potential. Type 1 are described as a thin fracture line with no evidence of intramedullary sclerosis, type 2 is characterised by a wider break line with clear evidence of sclerosis and the complete filling of the medullary canal by sclerotic bone classes as type 3. Based upon the morphology and position of the fracture it is classified in the Stewart classification system (Figure 3) [5].

\section{Virtual fracture clinic}

In 2016 there were 23.57 million attendances in England's A\&E departments out of which approximately 6 million were type 3 attendees which are minor injuries that can be treated by doctor-led or nurse-led teams in routine services [6]. Once the patient has been discharged from $A \& E$ they are referred to the required speciality, in the case of fractures they are sent to the virtual fracture clinic. This is different to the traditional clinic setting and uses fewer members of staff, resources and money. During the clinic, an orthopaedic nurse and consultant will discuss the patient's history and radiographs in the patient's absence and decide a management plan. The plan created is to either discharge the patient with an informative phone call timely or invite them into the clinic to receive the supplementary specialist care that is required (Table 1). A survey done in a district general hospital in

${ }^{\star}$ Correspondence to: Mohamad Alqubaisi, University Hospital of South Manchester NHS Foundation Trust, UK, E-mail: mohamad.alqubaisi@mft.nhs.uk

Received: September 24, 2018; Accepted: October 12, 2018; Published: October 16,2018 


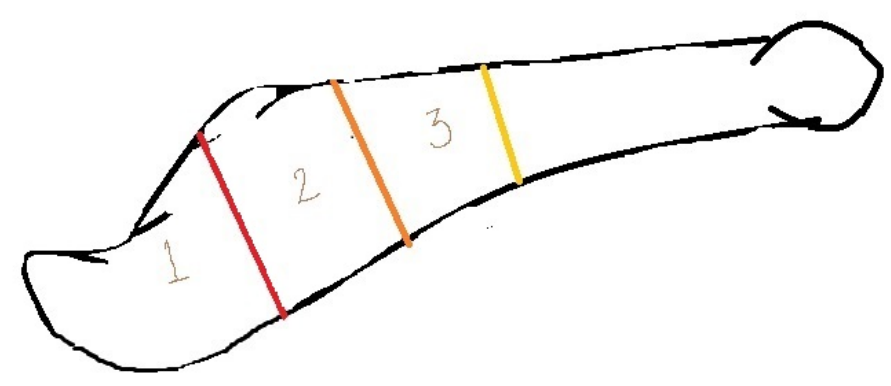

Figure 1. An illustration to represent zone 1,2 and 3 of the Dameron-Lawrence-Bofte classification [3]

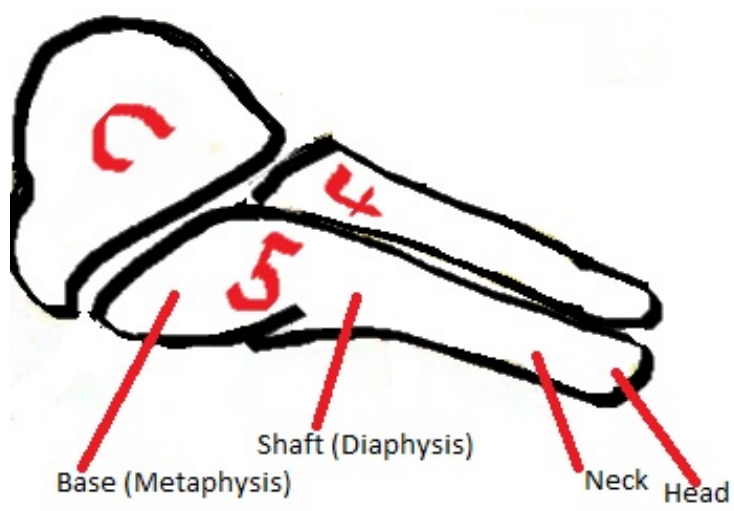

Figure 2. The fifth metatarsal's anatomical division [4]

Table 1. The current protocol for managing a fifth metatarsal fracture in the virtual fracture clinic [9]

\begin{tabular}{|l|l|l|l|}
\hline Diagnosis & Initial Treatment & A\&E Management & VFC action \\
\hline $\begin{array}{l}\text { Undisplaced 5 } \\
\text { Fracture }\end{array}$ & Moon boot & Discharge & \\
\hline Displaced & $\begin{array}{l}\text { Padded crepe, } \\
\text { analgesia \& crutches }\end{array}$ & Refer to VFC & $\begin{array}{l}\text { Next foot and ankle } \\
\text { clinic }\end{array}$ \\
\hline
\end{tabular}

Bury St Edmunds, England has shown that $97 \%$ felt that the phone call was useful and 95\% knew how to ask for help where needed [7].

A recent evaluation into the virtual fracture clinic at UHSM has shown that there has been at least a $25 \%$ reduction in the amount of face-to-face consultations. This has meant that improvements have been seen in 72 hours review targets and a decline in the number of cases that attend the fracture clinic. On average in each clinic per week about 6.7 patients worth of resources, staff time and cost has been saved [8].

\section{Anatomy}

The foot is comprised of 26 bones of which the fifth metatarsal is one of the most mobile and important in determining the shape of the foot. The various extrinsic and intrinsic muscle attachments enable these characteristics; hence many athletes and dancers sustain this fracture. Fifth metatarsal fractures can occur due to long standing pressure over time and these are known as stress fractures; categorised as Zone 3 [9]. Alternatively, they can manifest because of sudden trauma [10]. The nutrient artery to the fifth metatarsal enters the bone at the medial third and courses through the medial cortex before distributing into the shorter branches. There is a wealth of short metaphyseal vessels at the terminals of the bone. Thus, injuries to the proximal diaphysis are likely to injure and impair the blood supply to this area, hence why it is explained as a watershed in blood supply at that junction [11].

\section{Treatment}

Zone 1 fractures have shown to have an excellent prognosis and thus conservative methods of treatment are suggested. Hence these patients should not require many follow up appointments as they are not likely to suffer from complications [12]. It is well known that the metaphyseal-diaphyseal junction is a watershed area for blood supply thus injuries within this area (zone 2 and zone 3), are more prone to complications in their recovery for example delayed union or nonunion. Therefore, it is vital to ensure you have adequate clinical history to analyse the patient as a whole [13].

The Torg classification provides a better indication for management. Type 1 fractures take anywhere between 3-12 weeks to heal if managed with non-weight bearing treatment as per recommendations. Type 2 and 3 are more complicated due to the anatomy; in some cases type 2 can be managed conservatively whereas for other cases it may be more appropriate to consider surgery. Type 3 always requires operating as it is more likely that there will be complications to the metatarsals' recovery. However, it is important to acknowledge other factors that can have an effect on recovery time such as diet, activity levels and past medical history [13].

The data collected from this study has portrayed the virtual discharge and recall rate of the fracture in different classification systems. Additionally, the study demonstrates the number of patients that required the different management approaches (Figure 3 ).

\section{Metatarsus adductus}

Metatarsus Adductus is a common foot deformity that causes the distal half of the foot to turn medially and is commonly associated with an increased risk of stress fractures. It is due to the higher organic and mechanical stresses on the lateral metatarsals. In most cases conservative management has been found to be the most beneficial; however surgical restoration can be used in cases where the fundamental malformation is greater [14].

\section{Athletes}

A study conducted in 2015 focused on how athletes require different treatment compared to those that lead a relatively more sedentary lifestyle. Non-operative techniques in treating athletes can take as long as 20 weeks, this is not a feasible amount of time for them. Consequently, Japjec et al. designed a further classification for their study: Metaphyseal (zone 1 and majority of zone 2) and Meta-diaphyseal fracture (remnants of zone 2 and zone 3 ). The exact boundaries of this classification system are uncertain and require further research.

\begin{tabular}{|c|c|c|c|c|}
$\begin{array}{c}\text { Type 1: Extra- } \\
\text { articular } \\
\text { fracture between } \\
\text { the metatarsal } \\
\text { base and } \\
\text { diaphysis }\end{array}$ & $\begin{array}{c}\text { Type 2: Intra- } \\
\text { articular } \\
\text { fracture of } \\
\text { the metatarsal } \\
\text { base }\end{array}$ & $\begin{array}{c}\text { Type 3: } \\
\text { Avulsion } \\
\text { fracture }\end{array}$ & $\begin{array}{c}\text { Type 4: } \\
\text { Comminute } \\
\text { fractures with } \\
\text { intra-articular } \\
\text { extension }\end{array}$ & $\begin{array}{c}\text { Type 5: Partial } \\
\text { avulsion of the } \\
\text { metatarsal base } \\
\text { with or without } \\
\text { a fracture }\end{array}$ \\
\hline
\end{tabular}

Figure 3. Visual representation of the Stewart classification [5] 
Nonetheless the first would be treated using non-operative techniques and the latter with surgical fixation. Based on all of this information and the frequency of these fractures in athletes, this particular study had concluded that surgical treatments should be first line treatment for athletes with zone 2 and 3 proximal metatarsal fractures [15].

\section{Methods}

UHSM NHS Foundation trust is a teaching hospital that provides numerous amounts of services, including specialist tertiary services, to the community for patients of all ages and socio-economic background. A recent addition to this trust is the Virtual Fracture Clinic, this was started in August 2015 [16]. Audit approval was required and obtained from the trust and the University of Manchester to perform this study; on the other hand as this is a clinical audit it was not necessary to receive an approval from the research ethics committee.

All patients that presented to the VFC with a first presentation of fifth metatarsal fractures between August 2015 and March 2017 were identified. Data was extracted from the medical records in the archives and the Electronic Patient Record database on the patient demographics, details of their fracture and management. This was then complied into a table (Table 2).

Some patients from the VFC data were excluded as they had not been treated at UHSM, despite first presentation at its' A\&E department. In addition, some patients' records were not available, or records were lost therefore these were also excluded. If a patient had presented with a fifth metatarsal fracture but had been operated on previously for such e.g. ORIF, K-wires, they were also excluded. Following through with this, the total number of clinical cases identified was 270 .

Once the initial data had been collated, each fracture was classified in the different systems. First the fractures were separated based on their anatomical location and then categorised into the Dameron-LawrenceBofte classification, Torg classification and the Stewart classification (Figure 4). The information extracted from the management of the patients was: the type of treatment received, if advanced imaging was required, number of weeks from initial presentation till surgery if required and the total treatment period (weeks from initial presentation till discharge).

In some patients non-union and delayed union was recorded in the clinic letters however in those that it was not, non-union was determined if the x-rays at 4-6weeks showed little callus formation and clinical symptoms were present. As a typical fifth metatarsal fracture with conservative treatment takes 12 weeks to heal, any images and reports that depicted union after this point was classed as a delayed union. If the data for the patient was not available to calculate this, they were excluded at this point.

Table 2. Data collection table displaying the variables

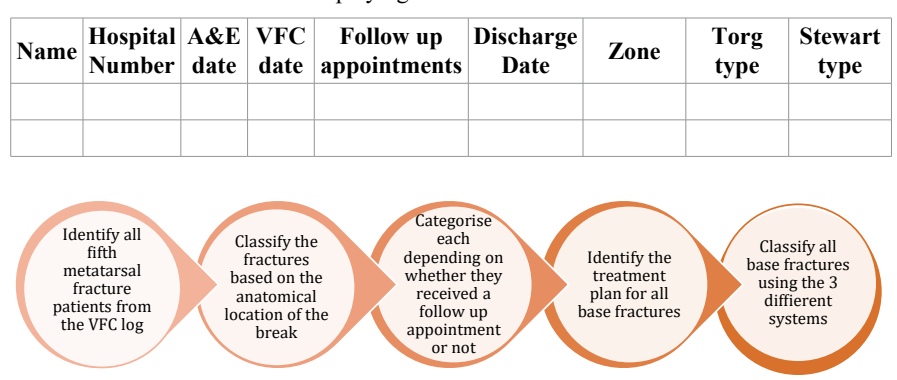

Figure 4. Summary of the method used
Using statistics software, the variables in the data collected was cross-tabulated to quantify the outcomes of the fractures and their frequencies. It allowed analysing the correlation between the outcomes and the classification systems. This software was useful in providing means to calculate the averages for example the average number of weeks till discharge per fracture type (Figure 4).

\section{Results}

270 patients were referred to VFC by A\&E over the period of August 2015-March 2017, 53.3\% (144/270) of these were base fractures, $28.6 \%$ $(77 / 270)$ had non-base fractures and the remaining $18.1 \%(49 / 270)$ did not have a fracture.

\section{Classifications}

Using Dameron-Lawrence-Bofte classification, 107 patients were classed as Zone 1, 31 in Zone 2 and 6 in Zone 3.

Zone 1: In Zone 1 fractures, 68.2\% (73/107) patients were discharged virtually. Out of the remaining $31.8 \%$ (34/107), only one patient required surgical intervention - open reduction internal fixation (ORIF) with $\mathrm{k}$-wires. This gives zone 1 fractures an operating chance of $2.9 \%$ (1/34). 3 patients underwent advanced imaging tests such as computed tomography (CT) or magnetic resonance imaging (MRI), 2 of these patients were referred to other services and the other was treated conservatively. 31 of the Zone 1 fractures were managed conservatively. This means there is a $91.2 \%$ (31/34) likeliness of Zone 1 fractures being treated conservatively.

It has been demonstrated that $28.6 \%(2 / 7)$ of non-union and $57.1 \%$ $(4 / 7)$ of delayed union fractures occur in Zone 1 (Table 3).

Zone 2: There were 31 Zone 2 fractures identified, out of which $25.8 \%$ were discharged virtually thus a recall rate of $74.2 \%(23 / 31)$. Zone 2 patients were classified using the Torg Classification too (Figure 5 ). Of the 23 patients that were recalled, 3 underwent ORIF, out of whom 1 patient had a CT scan before consenting and was a Torg type 3 (Table 3).

The other 2 patients were both Torg type 2 . The reminder 20 patients were managed conservatively; this means that there is an $87.0 \%(20 / 23)$ chance of Zone 2 fractures being managed with conservative treatment.

It has been demonstrated that $57.1 \%(4 / 7)$ of non-union and $42.8 \%$ $(3 / 7)$ of delayed union fractures occur in Zone 2. All of the delayed unions in this zone were Torg Type 2. 75\% (3/4) of the non-unions were Torg type 2 and the residual $25 \%(1 / 4)$ is a Torg Type 3 .

Zone 3: There were 6 Zone 3 fractures identified, out of which $16.7 \%(1 / 6)$ were discharged virtually thus a recall rate of $83.3 \%(5 / 6)$. Zone 3 patients were classified using the Torg Classification too. Of the 5 patients that were recalled, 0 underwent ORIF, and only 1 patient had a CT scan but went on to be managed supportively. Therefore all 5 patients were treated conservatively; this means that there is an $83.3 \%$ (5/6) chance of Zone 3 fractures being managed in this way. It has been demonstrated that $14.3 \%$ (1/7) of non-union fractures occur in Zone 3, there were not any cases of delayed union (Table 3 ).

Stewart classification: The 144 patients with basal fifth metatarsal fractures were classified using the Stewart Classification. The most common type of fracture was type 5 with $31.9 \%(46 / 144)$ fractures. Type 1 had $31.3 \%$ (45/144) fractures; type 2 had $11.1 \%(16 / 144)$ fractures, type 3 had 19.4\% (28/144) and type 4 had 6.3\% (9/144) fractures.

Type 2 were the most likely to achieve operative management and type 3 and 5 were the least (Figure 6). 
Comparing the Stewart classification and Dameron-LawrenceBofte classification, there does not appear to be a correlation between the zones and the types of fractures. Most of Type 5 fractures are Zone 1 but type 3 is also predominantly zone 1 (Figure 7).

Table 3. Summary of results

\begin{tabular}{|l|l|l|l|l|}
\hline \multicolumn{2}{|l|}{} & Zone 1 & Zone 2 & Zone 3 \\
\hline Patients classified & $74.3 \%(107 / 144)$ & $21.5 \%(31 / 144)$ & $4.2 \%(6 / 144)$ \\
\hline $\begin{array}{l}\text { Number of Follow up } \\
\text { appointments }\end{array}$ & Maximum & 16 & 9 & 2 \\
\cline { 2 - 5 } & Minimum & 1 & 1 & 1 \\
\cline { 2 - 5 } & Mean & 1.5 & 1.9 & 1 \\
\hline $\begin{array}{l}\text { Number of weeks till } \\
\text { Discharge }\end{array}$ & Maximum & 23 & 51 & 11 \\
\hline & Minimum & 1 & 1 & 5 \\
\hline Patients that required advanced imaging & $60.0 \%(3 / 5)$ & 11 & 7 \\
\hline $\begin{array}{l}\text { Patients that required operative } \\
\text { treatments }\end{array}$ & $25.0 \%(1 / 4)$ & $75.0 \%(3 / 4)$ & 0 \\
\hline $\begin{array}{l}\text { Patients managed with conservative } \\
\text { treatment }\end{array}$ & $56.9 \%(33 / 58)$ & $34.5 \%(20 / 58)$ & $8.6 \%(5 / 58)$ \\
\hline \multicolumn{2}{|l|}{ Patients that developed Non-union } & $28.6 \%(2 / 7)$ & $57.1 \%(4 / 7)$ & $14.3 \%(1 / 7)$ \\
\hline Patients with delayed union & $57.1 \%(4 / 7)$ & $42.9 \%(3 / 7)$ & 0 \\
\hline
\end{tabular}

\section{A bar chart to demonstrate the 14 number of Zone 2 patients, of each Torg type, against the management
they received

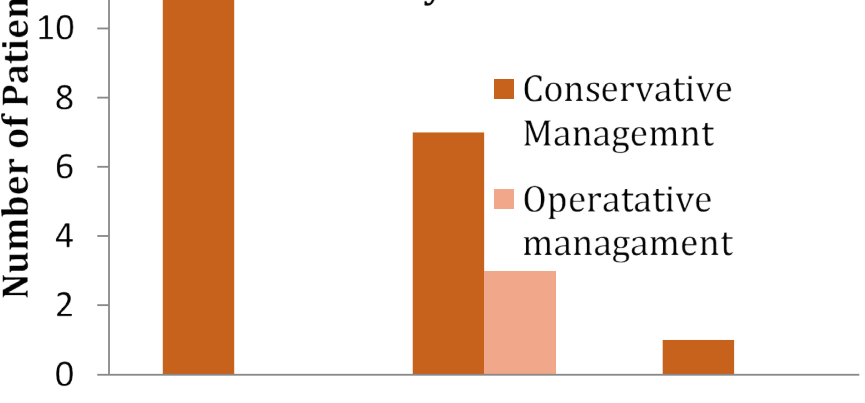 \\ Torg type 1 Torg Type 2 Torg Type 3 The Torg Classification types}

Figure 5. Zone 2 fractures relationship with Torg classification and the management

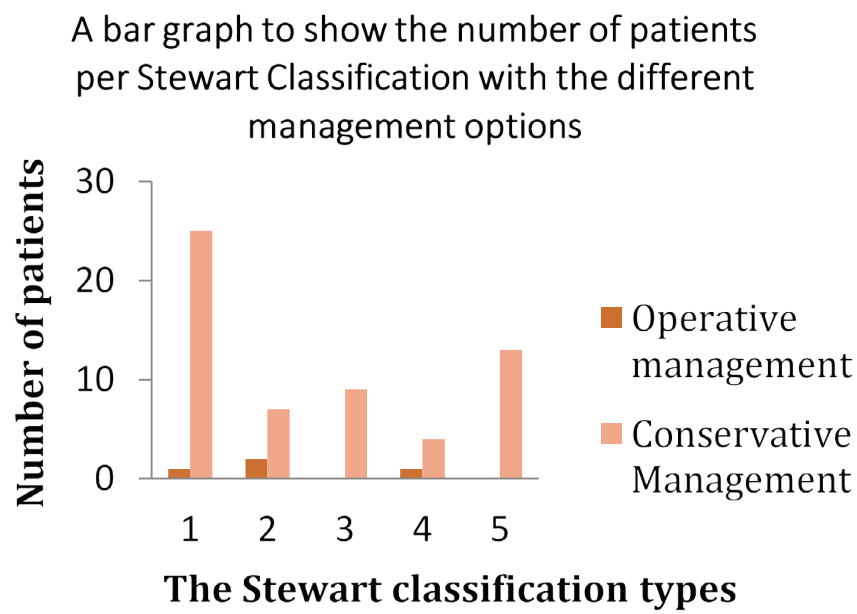

Figure 6. The distribution of management over the Stewart classification

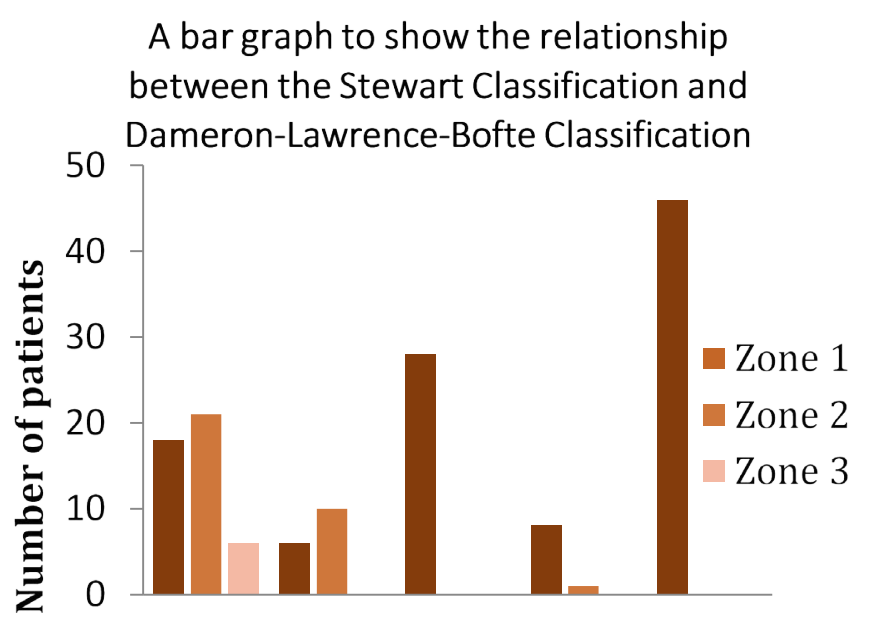

Type 1 Type 2 Type 3 Type 4 Type 5 The Stewart Classification

Figure 7. Displaying the relationship between the Stewart classification and DameronLawrence-Bofte classifications

\section{Follow up appointments}

From the 270 patients discussed in this study, 116 were offered follow up appointments in the clinic; this is a follow up rate of $43.0 \%$ in general. The follow up rate is calculated by the total number of follow up appointments for the category divided by the total number of fractures in that category. Non-basal fractures had a follow up rate of $44.2 \%$ (34/77), basal fractures had a follow up rate of $43.1 \%(62 / 144)$ and the patients without a fracture reported had a recall rate of $40.8 \%$ (20/49). Initially there doesn't appear to be a significant difference in the allocation of follow up appointments. However, when looking at the classified fractures it appears that on average Zone 2 fractures have the highest follow up rate and have the longest treatment journey.

\section{Discussion}

The results obtained in this study indicate that the virtual fracture clinic is a good platform to provide efficient and quality care. Patients appear to be satisfied with the service and the information they are provided with. There is a reduction in the amount of resources used for the patient's appointments such as plaster material - the plaster that was applied at A\&E would be removed for the consultation and then reapplied as the patient left the clinic. Patients need to arrange for transportation and accommodate their commitments around their appointment times, for a discharge appointment. This wastes the patient's time as well as the clinics. Each clinic slot can last anywhere between 10-25 minutes, it is valuable time of the consultants and has a significant impact on the cost. That clinical slot could have been used by a more serious fracture; this would allow for those fracture's treatment journey to be shortened. Noticeably there is a reduction in patient's visits by 6.7 per clinic per week as shown by a study done at UHSM [8].

\section{Zone 1}

Dameron-Lawrence-Bofte classification and Torg classification are of good prognostic value in determining the treatment length. Zone 1 fractures are the most frequent and have been treated with conservative treatment predominantly. Only one of the 34 patients that had received a follow up appointment for this category had required surgical intervention in the form of ORIF. This patient is a young active male, who had shown to have hind foot valgus. Therefore 2 
weeks after his initial fracture he was consented for ORIF with k-wires. Having had his operation, he was reviewed at 5 weeks at which point his wound was healthy, K-wires were removed, and he was showing signs of clinical improvement. 4 weeks after this he was discharges as his metatarsal had united. As discussed in the Introduction section 1.5 Athletes, the activity level of the patient has an effect on the recovery, the more active a being the more intense the treatment regimen should be [15]. This supports the idea of discharging zone 1 fractures virtually. However, this should be taken with precaution, as further investigation into the factors that can affect the bone healing will also need to be acknowledged.

\section{Zone 2}

Zone 2 is at the metaphysis and diaphysis junction, this is a watershed area for blood loss and thus injury to this requires more specialist care. One of these patients is a 55-year-old female, whom has still not been discharged from the clinic. 14weeks after her initial fracture it was arranged for her to have a CT scan which showed a tendency towards metatarsus adductus. As discussed earlier in the introduction section 1.4 metatarsus adductus, this is one of the indicators for being predisposed to attaining fifth metatarsal fractures [14]. Earlier scans had shown for her to have foot pes planus and looking further into her clinical history, it was believed that she had a fracture in 2011 which may not have healed completely and over time it has become worse. Looking back now, the consultant that reviewed her x-ray believes it may have been a Jones fracture or stress fracture which would explain why without adequate treatment it has deteriorated. Her last review appointment was at 12 weeks post - operation which showed signs of callus formation and so has been arranged for another appointment in 12 weeks to ensure the fracture has completely healed.

A 36 year old female who obtained a zone 2 fracture was placed on the ORIF procedure list suggesting that she had a delayed union, non-union complication for which she required operative treatment. Initially she had been discharged from the clinic but she was in a lot of pain and having difficulty walking so arranged to come back to the clinic. A fifth metatarsal fracture usually takes about 3-12 weeks to heal, as his fracture had had the upper limit of 12 weeks and the patient was still showing clinical symptoms she was placed on the ORIF procedure list with bone grafts. It was not until 10weeks post -operatively that union had been reached. This signifies the importance of clinical presentation as well as the radiographic results as mentioned in the introduction in section 1.2 Treatment [13].

Another patient that received that received ORIF for her zone 2 fracture demonstrated non-union, therefore was consented for the procedure 2 weeks after her initial presentation. However, her postoperative recovery was longer. 9weeks post operatively she had an $\mathrm{x}$-ray which was still not convincing of union and so was continued to be followed up, it wasn't till 6 weeks after this that she showed signs of union and was discharged. Thus, making her post-operative recovery time as 15 weeks, suggesting a delayed union post-operative [17].

\section{Torg classification}

Looking at the patients that were in Zone 2 and classified in the Torg Classification, it shows that Zone 2 Torg Type 2 were the most frequent to receive operative treatment. This agrees with the literature as this is an area of large blood supply and a lower healing potential [18].

One patient whom had a Zone 2 Torg type 3 fracture was thought to have a "Jones" fracture when viewed at 6weeks. Callus formation was seen but the fracture was not healing at a steady rate. As he is a non-smoker, works from home and compliant, it was thought that he should be given an opportunity to manage without surgery. $\mathrm{He}$ was appointed another clinic visit 6 weeks later but he cancelled this appointment, suggesting that the fracture had healed. This relates to the background information related on the patient and the bone health [19]. Smoking, activity levels and compliancy are all some of the most important factors in determining bone health [19].

\section{Zone 3}

Patients in zone 3 were all treated conservatively, a patient showed stress fracture non-union, with bridging callus and clinically tenderness over the base of the fifth metatarsal. It was arranged for him to have a CT scan performed in 2 months' time. This scan showed that he had near complete cortical union and improvement clinically and so was discharged. As he had a fracture previously that had been treated conservatively over 3 months ago, initial management focused on observations and imaging rather than surgical intervention. This is important as it allows for a deeper understanding of the cortical strength, due to the anatomical intricacies and healing potential of this region [17].

\section{Stewart classification}

The Stewart classification has been useful in understanding the effects of the tuberosity fractures such as the intra-articular fracture (type 2 ) and the avulsion fractures (type 3 or 5 ). However, upon comparing and contrasting these results against the Dameron-Lawrence-Bofte classification, it has shown that it is not a good indicator of prognosis but rather a system to understand the effects of the fracture [5].

\section{Virtual fracture clinic}

This study demonstrates that the virtual fracture clinic is safe, cost and time efficient approach to managing fifth metatarsal fractures. It has shown to reduce the number of follow up appointments overall, however this can be reduced further if there is a better protocol to follow to classify the fractures before deciding the management plan. In clinical practice it is important to consider how the classification of these fractures can be included in the protocol.

Ideally if the radiographers could classify the fractures when interpreting the images, it would become easier for the team at VFC. If this were to occur, there would be the need for more education of the radiographers of these classification systems. This could be delivered in the form of a poster with clear depictions of what each class should appear as. Alternatively, a new protocol could be created allowing for the consultant to classify the fracture in the VFC.

In order to do this, there would need to be an agreement between the consultants of the orthopaedic department as to what classification should be used. Dameron-Lawrence-Bofte's classification allows for the fractures to be assorted dependant on location; together with the Torg classification it would allow for a better understanding of the healing potential and thus management. From this study, it has been found that the Stewart classification does not differentiate or provide more guidance than Dameron-Lawrence-Bofte's and Torg's classifications combined. These systems have allowed for categorisation based on location in relation to blood supply and healing potential. There is also more literature and research in to this, which allows for a better understanding.

\section{Strengths \& limitations}

There is a lot of literature available on the classification of the fifth metatarsal fractures, this meant that the fundamental knowledge 
needed for this audit was easily accessible. It was not an expensive study and did not require any new resources. The method is straight forward and so can easily be replicated. Even though it was a time-consuming process to identify the patients manually from all of the patients in the virtual fracture clinic and analysing the data, this project was completed within 2 months. This study has identified a large sample size of 270 patients this means that the data and the results obtained are representative of the population discussed.

Nevertheless, as this sample size only looks at specific fractures, not all cases are discussed in depth. The patients looked at were not sampled for the different variables and so this can have an effect on the outcomes of each individual case [20]. For example, the older a patient is the more likely they're bones are to break as they may be osteopenic and/or suffer from osteoporosis. Osteoporosis becomes more prevalent with age especially in patients with renal impairment, as this can have an adverse effect on the bone mineral density resulting in weaker bones that are more easily broken and fractured [21].

The inverse is also true, growing active children and adolescents, may have apophysis of the fifth metatarsal base. It can disappear spontaneously once the child has completed growing. On a radiograph it appears as an outward growth of the base that has been displaced [22] Islein's disease, it is a self-limiting disease which presents with pain in the proximal fifth metatarsal region and thus is often misdiagnosed as a proximal metatarsal fracture. It is due to the repetitive burden on the growth plate. Treatment for this is also similar to proximal fifth metatarsal fractures in that it can be managed conservatively but nonunion may occur which can result in pain lasting till early adulthood [23].

In addition to age and bone density, other factors that may have an influence include diet and lifestyle for example smoking and heavy alcohol use has been correlated with a reduction in bone mass and an increase fracture risk [19]. Another study has shown that diabetes also has an effect on one healing potential due to the demineralizing effects diabetes has on the bones specifically the metatarsal bones [24].

This study design did not take in account any of these confounding variables that may have had an impact on the reasoning behind arranging a follow up appointment. This information was also not easily accessible on EPR database or in the VFC log; to enhance this study further going through each patient record to identify all factors would be recommended.

In this study a medical student reviewed the X-rays, whom was not trained in reading foot and ankle $\mathrm{x}$-rays, therefore used the classifications and imagery available from other studies to help categories. This raises questions about the reliability of the data, especially highlighting kappa coefficient. The kappa statistic looks at the inter-rater reliability and if the variables that have been measured are representative [25]. However, this cannot be done for this study as there was only one investigator and thus there is a higher chance of investigator bias meaning the results may be skewed [26]. It is therefore recommended to re-audit this project to ensure the statistics achieved are reliable.

\section{Conclusion}

The virtual fracture currently has helped to reduce the number of clinical visits without affecting the quality of care. However, the protocol currently used in VFC can be enhanced to include classification of these fractures, this will provide a clearer treatment regimen. Zone 1 and 3 fractures are most likely to reach union with supportive management. Hence, they can be discharged virtually, if the fracture has good healing potential and no risk factors that could delay the union are present. Zone 2 fractures are more likely to require operative intervention, as they are the most likely to develop nonunion. Thus, should always receive a follow up appointment at 6 weeks. The Torg classification would allow further guidance on this as Torg type 2 and type 3 almost always need surgery for full restoration of the fracture. The extenuating factors that could affect healing potential were not explored in this cohort of patients. Therefor more research into this will allow a better understanding on the likelihood of delayed union, non-union complication for each zone.

\section{References}

1. Jones R (1902) I. Fracture of the base of the fifth metatarsal bone by indirect violence. Ann Surg 35: 697-700. [Crossref]

2. Carp L (1927) Fracture of the fifth metatarsal bone: with special reference to delayed union. Ann Surg 86: 308-320. [Crossref]

3. Lawrence SJ, Botte MJ (1993) Jones' fractures and related fractures of the proximal fifth metatarsal. Foot Ankle 14: 358-365. [Crossref]

4. Batold S (2016) LBG Medical.

5. Stewart IM (1960) Jones's fracture: fracture of base of fifth metatarsal. Clin Orthop 16: 190-198. [Crossref]

6. Steele P (2017) Quarterly attendances \& emergency admission monthly statistics. NHS and independent sector.

7. Singh A, Nicholai P, Porteus M, Deakin S (2014) The virtual fracture clinic pathway-A novel. Joint 66.

8. Booth S, Pillai A, Butler D. An evaluation of a virtual fracture clinic pilot.

9. Fracture Clinic, University Hospital of South Manchester. Virtual Fracture Clinic protocol 2015.

10. Johnson J (2015) American Orthopaedic foot and ankle society.

11. Dameron TB Jr (1995) Fractures of the proximal fifth metatarsal: selecting the best treatment option. J Am Acad Orthop Surg 3: 110-114. [Crossref]

12. Ferguson KB, McGlynn J, Jenkins P, Madeley NJ, Kumar CS, et al. (2015) Fifth metatarsal fractures - Is routine follow-up necessary? Injury 46: 1664-1668. [Crossref]

13. Zwitser EW, Breederveld RS (2010) Fractures of the fifth metatarsal; diagnosis and treatment. Injury 41: 555-562. [Crossref]

14. Lemos DW, Manoli A (2011) Stress fractures in patients with metatrsus adductus.

15. Japjec M, Staresinic M, Starjacki M, Zgaljardic I, Stivicic J, et al. (2015) Treatment of proximal fifth metatarsal bone fractures in athletes. Injury 46 Suppl 6: S134-136. [Crossref]

16. University Hospital of South Manchester NHS Foundation trust. Annual reports and accounts. April 2015- March 2016.

17. Bowes J, Buckley R (2016) Fifth metatarsal fractures and current treatment. World $J$ Orthop 7: 793-800. [Crossref]

18. Lehman RC, Torg JS, Pavlov H, DeLee JC (1987) Fractures of the base of the fifth metatarsal distal to the tuberosity: a review. Foot Ankle 7: 245-252. [Crossref]

19. Determinants of Bone health. In Bone Health and Osteoporosis: A Report of the Surgeon General, Rockville 2004.

20. Biau DJ, Kernéis S, Porcher R (2008) Statistics in brief: the importance of sample size in the planning and interpretation of medical research. Clin Orthop Relat Res 466 : 2282-2288. [Crossref]

21. Klawansky S, Komaroff E, Cavanaugh PF Jr, Mitchell DY, Gordon MJ, et al. (2003) Relationship between age, renal function and bone mineral density. Osteoporos Int 14 : 570-576. [Crossref]

22. Schwartz B, Jay M, Schohoenaus HD (1991) Apophysitis of the fifth metatarsal base. Iselin's disease. J Am Podiatr Med Assoc 81: 128-130. [Crossref]

23. Canale ST, Williams KD (1992) Iselin's disease. J Pediatr Orthop 12: 90-93. [Crossref]

24. Fleischli J, Laughlin T, Lavery L, Shah B, Lanctot D et al. (1998) The effects of diabetes mellitus on the material properies of human metatarsal bones. The Jounal of Foot and Ankle surgery 37: 195-198. 
Robinson A (2018) How are fifth metatarsal fractures managed by the virtual fracture clinic?

25. McHugh M (2012) Interrater reliability: the kappa statistic. Biochemia Medica 22: 276-282.
26. Wynder E (1994) Investigator bias and interviewer biad: the problem of reporting systematic error in epidemiology. J Clin Epidemiol 47: 825-827. [Crossref]

Copyright: $(02018$ Mirza Z. This is an open-access article distributed under the terms of the Creative Commons Attribution License, which permits unrestricted use, distribution, and reproduction in any medium, provided the original author and source are credited. 